\title{
Optimal economic dispatch of power generation solution using lightning search algorithm
}

\author{
Murad Yahya Nassar', Mohd Noor Abdullah', Asif Ahmed Rahimoon ${ }^{3}$ \\ ${ }^{1,3}$ Green and Sustainable Energy (GSEnergy) Focus Group, Faculty of Electrical and Electronic Engineering, Universiti \\ Tun Hussein Onn Malaysia (UTHM), Malaysia \\ ${ }^{2}$ Department of Electrical Engineering, Universiti Tun Hussein Onn Malaysia, Malaysia
}

\begin{tabular}{l} 
Article Info \\
\hline Article history: \\
Received Feb 2, 2020 \\
Revised Apr 18, 2020 \\
Accepted May 1, 2020 \\
\hline
\end{tabular}

\section{Keywords:}

Economic dispatch

Power system

Transmission losses

Valve-points loading

\begin{abstract}
Economic dispatch (ED) is the power demand allocating process for the committed units at minimum generation cost while satisfying system and operational constraints. Increasing cost of fuel price and electricity demand can increase the cost of thermal power generation. Therefore, robust and efficient optimization algorithm is required to determine the optimal solution for ED problem in power system operation and planning. In this paper the lightning search algorithm (LSA) is proposed to solve the ED problem. The system constraints such as power balance, generator limits, system transmission losses and valve-points effects (VPE) are considered in this paper. To verify the effectiveness of LSA in terms of convergence characteristic, robustness, simulation time and solution quality, the two case studies consists of 6 and 13 units have been tested. The simulation results show that the LSA can provide optimal cost than many methods reported in literature. Therefore, it has potential to solve many optimization problems in power dispatch and power system applications.
\end{abstract}

This is an open access article under the $\underline{C C B Y-S A}$ license.

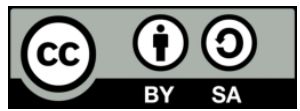

\section{Corresponding Author:}

Mohd Noor Abdullah,

Green and Sustainable Energy (GSEnergy) Focus Group,

Faculty of Electrical and Electronic Engineering,

Universiti Tun Hussein Onn Malaysia,

Parit Raja, 86000 Batu Pahat, Johor, Malaysia.

Email: mnoor@uthm.edu.my

\section{INTRODUCTION}

The economic dispatch (ED) is one of the optimization problems in power system operation and planning to allocate the shared power demand between the generating units. Thus, optimal power system operation is important in electrical networks to ensure the system can operate at minimal cost. Therefore, the aim of the ED is to minimize the total cost of generation and satisfy the system and operating constraints [1].

Various optimizations methods have been proposed and applied to solve ED problem over the latest decades and can be classified into two main categories such as classical method and heuristic method [2]. The classical methods such as newton's method [3], quadratic programming technique [4], interior point [5], lambda iteration method [6], evolutionary programming (EP) techniques [7] and dynamic programming [8] are widely used for solving convex and smooth cost function of ED problem. However, most of these methods have difficulty for solving nonconvex or nonsmooth problems. To solve this problem, the non-conventional or heuristic methods are developed to solve the complicated and highly nonconvex optimization problem. The optimization algorithms such as ant colony optimization (ACO) [9], artificial bee colony $(\mathrm{ABC})$ [10], firefly algorithm (FA) [11], particle swarm optimization (PSO) [12], teaching-learning- 
based optimization (TLBO) [13], genetic algorithm (GA) [14] and adaptive charged system search algorithm (ACSS) [15] have been solved the complex ED problem. The performance of these algorithms is better than classical optimization methods in many aspects for instance fast, robust and easy to adjust according to the problem. In some application, these algorithms are suffering from slow convergence rate, stuck at local solution and required proper parameter tuning to obtain optimal solution.

Furthermore, the hybrid method has been introduced by combining two or more algorithms in order to mitigate their weaknesses and use their strengths to provide better performance for solving optimization problems [16]. The proposed hybrid algorithms such as GA-PS-SQP [17], NM-FAPSO [18] and differential evolution algorithm-PSO [19] shows highly efficient technique to solve the ED problem. However, it required long computational time and complex programming since two or more algorithms are used.

Recently, a new nonconventional algorithm called the LSA algorithm [20] has never applied for solving ED problem. It was successfully used and implemented to optimize the different applications such as binary optimization [21], nuclear reactor controller [22], and Fuzzy Logic PV Inverter Controller [23]. It shows the LSA can provide superior performance over other algorithms. Therefore, this paper proposed LSA as a new approach for solving ED problems with VP. The performance of LSA has been compared with other common methods. The remaining of this paper is organized as follows: Section 2 provides the Mathematical formulation of ED problem considering VPE and losses, section 3 presents the LSA technique, Section 4 introduces the simulation results for the test system and its analysis.

\section{PROBLEM FORMULATION OF ECONOMIC DISPATCH (ED)}

\subsection{Objective function}

The main aim of any ED problem is minimizing the system operation cost in order to fulfill the power demand alongside the generator limits. The fuel cost function can be differentiated as a simplified cost function and modified function by including with VPE. The cost function of production units can be represented by a quadratic function as follows:

$$
\begin{aligned}
& F\left(P_{G}\right)=\sum_{i=1}^{N_{g}} F_{i}\left(P_{G i}\right) \\
& F_{i}\left(P_{G i}\right)=a_{\mathrm{i}} P_{G i}^{2}+b_{\mathrm{i}} P_{G i}+c_{\mathrm{i}}
\end{aligned}
$$

Considering VPE for ED problem in the fuel cost functions exhibit a bigger variation in the generating units with multi-valve steam turbines. The generated power will be changed when every steam valve closes or opens. In the curves of the heat ratio introduces ripples by VPE. The cost function of ED will add a sinusoidal term and it can be defined mathematically as:

$$
F_{i}\left(P_{i}\right)=\left(a_{i}+\mathrm{b}_{i} P_{G i}+c P_{G i}^{2}\right)+\left|e_{i} \sin \left(F_{i}\left(P_{G i m i n}-P_{G i}\right)\right)\right|
$$

where $F\left(P_{G}\right)$ is the total cost of production, $F\left(P_{G i}\right)$ is the unit function of fuel cost $i ; \alpha_{i}, b_{i}, c_{i}, e_{i}$, and $F_{i}$ are the unit coefficients of fuel cost $i$; $P_{G i}$ is the unit output $i$ of real power.

\subsection{System operation constraints}

It should satisfy the following system constraints when ED Optimization carried out. The constraints considered for this research are discussed in sections 2.2.1 and 2.2.2.

\subsubsection{Equality constraint}

The equality constraint is represented the power balance equation and it is used in the optimization to ensure that the total power generated must be equal to the power demand in the case without losses in the system can be written as:

$$
\sum_{i=1}^{n} P_{i}=P_{D}
$$

If consider power losses, total generated power must be equal power losses and power demand as follows: 


$$
\sum_{i=1}^{n} P_{i}=P_{L}+P_{D}
$$

where $P_{i}$ is the power generated, $P_{L}$ Indicates transmission loss, $P_{D}$ is the load demand and $n$ is the overall amount of the generating units. The power losses can be calculated as:

$$
P_{L}=\sum_{i=1}^{n} \sum_{i=1}^{n} P_{g i} B_{i j} P_{g i}+\sum_{i=1}^{n} B_{0 i} P_{g i}+B_{00}
$$

where $B_{i j}, B_{o i}$, and $B_{00}$ are to determine the loss coefficient matrix

\subsubsection{Inequality constraint}

A power limit constraint is used to ensure that the generating unit operates within the minimum and maximum limits. This constraint presented as follow:

$$
P_{i \min } \leq P_{i} \leq P_{i \max }
$$

where $P_{\text {imin }}$ and $P_{\text {imax }}$ are minimum and maximum generating power limits of $i^{\text {th }}$ generating units.

\section{LSA ALGORITHM}

The LSA algorithm is a new metaheuristic method developed in 2015 by [20]. This method can be used to optimize complex nonlinear problems. It derived from a natural lightning phenomenon as a step leader propagation mechanism [24].

Nearby the thundercloud region can be found oxygen and nitrogen and hydrogen molecules. During the water molecules are freezing within a thundercloud, parts of water molecules are unable to fit the ice structure. Thus, these molecules at high speeds will be ejected from the forming ice. Therefore, the hydrogen and oxygen atoms are detached and ejected randomly in different directions as projectiles. These projectiles travel over the atmosphere and start the ionization path over collision and transition into the step leader. In this proposed algorithm, every projectile will create a step leader or channel that represents the initial population size. The projectile concept in this algorithm is highly similar to "particle" used in PSO.

The projectiles are taken into consideration which is known as the fast particles involvement is recognized in the structure formation of the binary tree of the step leader. Also in the formation of concurrent of two leader tips at points of the fork instead of the conventional mechanism of step leader which utilizes the streamers concept [20]. There are three types of projectiles that are developed such as the transition projectiles to produce initial solutions, space projectile provides exploration for good solutions and the lead projectile performs exploitation. Figure 1 presents a flowchart of proposed LSA for solving ED problems.

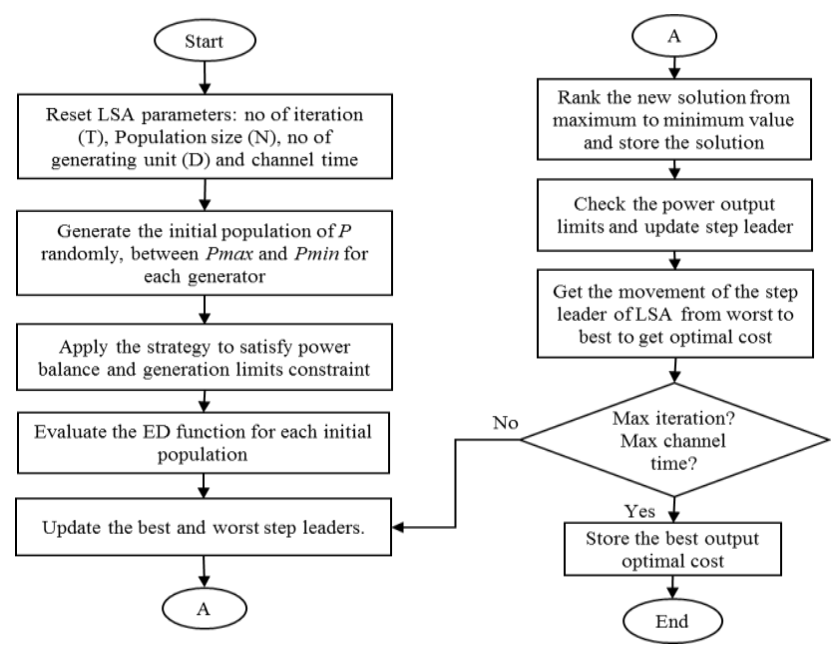

Figure 1. LSA Flowchart of proposed LSA for implementation on ED problem 


\subsubsection{Transition projectiles}

This projectile type is responsible for producing initial step leaders form the early stage because the transition projectile ejected from thunder in direction randomly. Therefore, by using a uniform probability distribution, this stage can be modeled in a random number. It can be described as follows:

$$
f\left(x^{T}\right)= \begin{cases}\frac{1}{b-a} & \text { for } a \leq x^{T} \leq b \\ 0 & \text { for } x<a \text { or } x^{T}>b\end{cases}
$$

where $x^{\mathrm{T}}$ represents the random number trying to give the solution or provide initial tip energy $E^{S} l$ of step leader $S^{l} i$ and the population of $N$ can be given as $S^{L}=\left[\mathrm{s}^{\mathrm{l}} 1, \mathrm{~s}^{\mathrm{l}} 2, \mathrm{~s} 3, \ldots, \mathrm{s}^{\mathrm{l}} \mathrm{N}\right]$, and $\mathrm{P}^{\mathrm{T}}=\left[\mathrm{p}^{\mathrm{T}} 1, \mathrm{p}^{\mathrm{T} 2}, \mathrm{p}^{\mathrm{T}} 3, \ldots, \mathrm{p}^{\mathrm{T} N}\right]$ for satisfying the dimension of the required solution.

\subsubsection{Space projectile}

Space projectile tries to explore the process in order to achieve the best position of leaders through ionizing the region of the old leaders. Therefore, the step leaders will be changed after the ionization of step leader tips tip in step+1. The space projectile $\mathrm{P}^{\mathrm{S}}$ location $=\left[\mathrm{p}^{\mathrm{S} 1}, \mathrm{p}^{\mathrm{S} 2}, \mathrm{p}^{\mathrm{S} 3}, ., \mathrm{p}^{\mathrm{SN}}\right]$ can be slightly designed as a random number produced from an exponential distribution with shaping parameter $\mu$. The following equation can be expressed as:

$$
f\left(x^{S}\right)= \begin{cases}\frac{1}{\mu} e^{-x^{S} / \mu} & \text { for } x^{S} \geq 0 \\ 0 & \text { for } x^{S} \leq 0\end{cases}
$$

where $\mathrm{x}^{\mathrm{S}}$ presents a random variable. The location or direction of space projectile can be clearly denoted in the equation and can be in charge of by shaping parameter $\mu$. The distance between space projectile $\mathrm{p}^{\mathrm{Si}}$ and lead projectile $\mathrm{p}^{\mathrm{L}}$ is taken by $\mu_{\mathrm{i}}$ for specific $\mathrm{p}^{\mathrm{Si}}$. According to this description, $\mathrm{p}^{\mathrm{Si}}$ position at step+1 is given as:

$$
P_{i_{-} \text {new }}^{S}=P_{i}^{S} \pm \exp \operatorname{rand}\left(\mu_{i}\right)
$$

where exprand is an exponential variable created randomly. When $\mathrm{p}^{\mathrm{Si}}$ has a negative value, then it should subtract the produced random number due to (10) that provides only positive values. Therefore, new position $\mathrm{p}^{\mathrm{Si}}$ new does not ensure stepped leader propagation until it can find a good solution.

\subsubsection{Lead projectile}

The lead projectile travels nearest to the ground and its associated projectile does not have enough potential for the large ionizing section in front of the leading tip. Therefore, it can obtain from normal distribution a modeled the lead projectile as a random number.

$$
f\left(x^{L}\right)=\frac{1}{\sigma \sqrt{2 \pi}} e^{-\left(x^{L}-\mu\right)^{2} / 2 \sigma^{2}}
$$

where $\mu$ is a shaping parameter to specify the present location of lead projectile $\mathrm{p}^{\mathrm{L}}$ and $\sigma$ is the scaling parameter that is responsible to exploit the projectile ability. Thus, $\mathrm{p}^{\mathrm{L}}, \sigma$, and $\mu$ are decreasing exponentially and progress to discover the best solution. Based on this idea, at step +1 the $\mathrm{p}^{\mathrm{L}}$ position can be written as:

$$
P_{\text {new }}^{L}=p^{L}+\operatorname{normrand}\left(\mu_{L}, \sigma_{L}\right)
$$

where normrand presents a random number produced by the distribution function. $p^{L}$ is an updated location of the lead projectile while $p^{L}$ new is the new location. In a similar way, if $p^{L}$ new does not get a good solution, then step leader will provide to a new location.

\section{SIMULATION RESULTS}

In this research, the LSA algorithm is applied to two different test systems as discussed in section 4.1.1-4.1.2, to validate the proposed algorithm for ED problems. In order to investigate the performance of 
the LSA algorithm, the achieved results have compared with different experimental results of the latest literature. The power balance and generator limits constraints are considered for all test systems. LSA technique has implemented in Matlab R2018b considering 6 and 13 units from the previous studies. The parameters used in the experimental study of LSA; 50 projectiles as the population size of the algorithm, the maximum number of the iteration is 500 and 50 multiple runs in every case study having 50 various initial solutions.

\subsubsection{Test case 1}

This test system has implemented for six thermal generating units using the proposed LSA approach. The demand for power in this test case is 283.4 MW and the transmission loss coefficient is given in [25]. The cost function with the VPE and losses are considered. Table 1 presents the comparisons of optimal results obtained by the LSA with existing approaches i.e. MSG-HS [26], PSO [26], and GA [25]. The total value of generation cost that can be achieved by LSA reached $925.122(\$ / \mathrm{h})$ is a bit lower and better than compared methods. It provides also other statistics results for LSA performance i.e. simulation time, best, average and the worst cost. The convergence and robustness of the LSA for minimizing cost is shown in Figure 2(a-b). Figure 2(a) it is observable that the LSA is trying to converge the cost function with the best iteration from $40^{\text {th }}-500^{\text {th }}$ for cost value. Moreover, Figure (b) illustrates the consistency of the optimal result after 50 runs with standard division around 12.02. It found that 31 runs can produce minimum cost results of about $925.122 \$ / \mathrm{hr}$.

Table 1. Comparison results for 6 units

\begin{tabular}{|c|c|c|c|c|}
\hline \multirow{2}{*}{ Units } & \multicolumn{4}{|c|}{ Power output (MW) } \\
\hline & LSA & MSG-HS [26] & PSO [26] & GA [25] \\
\hline P1 & 199.628 & 199.633 & 197.865 & 150.724 \\
\hline $\mathrm{P} 2$ & 20.074 & 20 & 50.337 & 60.870 \\
\hline P3 & 24.239 & 23.762 & 15 & 30.896 \\
\hline P4 & 20.073 & 18.393 & 10 & 14.214 \\
\hline P5 & 17.912 & 17.102 & 10 & 19.489 \\
\hline P6 & 12.506 & 15.692 & 10 & 15.915 \\
\hline The power generated (MW) & 294.37 & 924.583 & 295.758 & 292.11 \\
\hline Losses (MW) & 10.97 & 11.183 & 11.802 & 8.706 \\
\hline Best Cost $(\$ / \mathrm{hr})$ & 925.122 & 925.640 & 925.758 & 996.037 \\
\hline Average Cost $(\$ / \mathrm{hr})$ & 930.88 & 925.64 & 925.76 & - \\
\hline Worst Cost $(\$ / \mathrm{hr})$ & 962.959 & 928.6 & 928.6 & 1117.13 \\
\hline Power Demand & $283.4 \mathrm{MW}$ & 283.4 MW & 283.4 MW & $283.4 \mathrm{MW}$ \\
\hline Simulation time (s) & 1.285 & 0.621 & 0.353 & 0.578 \\
\hline
\end{tabular}

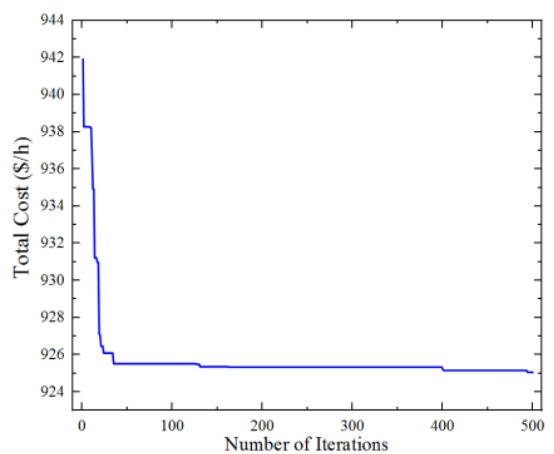

(a) Convergence characteristic

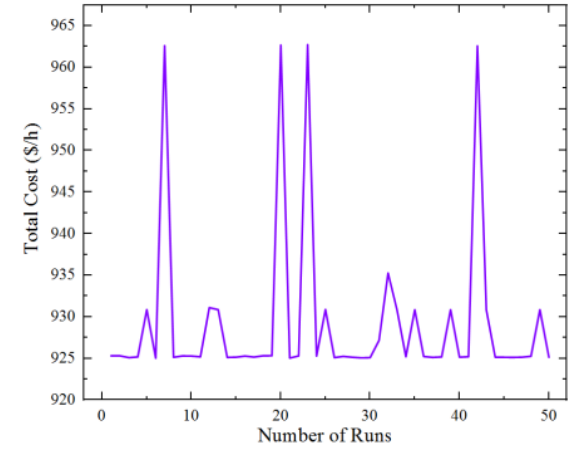

(b) Robustness of Cost

Figure 2. LSA performance for test case 1

\subsubsection{Test case 2}

A system of 13 generators considering VPE has examined with two different load demands i.e. 1800 MW without transmission losses and $2520 \mathrm{MW}$ with transmission losses. The input data for test case 2 with and without losses have taken from [1]. The obtained result from $1800 \mathrm{MW}$ load demand have compared with other algorithms as improved coordinated aggregation-based PSO (ICA PSO) [27], quantum-inspired particle swarm optimization (QPSO) [28], chaotic differential evolution and sequential quadratic programming (DEC SQP) [29] as shown in Table 2. While, the test results of $2520 \mathrm{MW}$ load demand 
considering losses are also compared with existing solutions i.e. OIWO [1], SDE [30], ORCCRO [31] as shown in continuous para of Table 2. It is observable that the minimum cost obtained using LSA is about 17963.95 (\$/hr) for without loss test and the lowest cost of about $24507.66(\$ / \mathrm{hr})$ for losses test. It also provides other statistics results i.e. simulation time, best, average and the worst cost for LSA in comparison with losses test system literature.

The convergence and robustness of the LSA for minimizing cost is shown in Figure 3(a-d). Figure 3(a) represents the convergence characteristics of the $1800 \mathrm{MW}$ load demand using LSA. The cost value has converged for the cost function with the best iteration from $150^{\text {th }}-500^{\text {th }}$. Moreover, Figure $3(\mathrm{~b})$ illustrates the consistency of the optimal result after 50 runs with a standard division of 50.86. Similarly, Figure 3(c) for $2520 \mathrm{MW}$ load demand the converged value tor cost function with the best iteration is $180^{\text {th }}$ $500^{\text {th }}$ and the robustness of load demand with losses is shown in Figure 3(d).

Table 2. Results comparison for test case 2

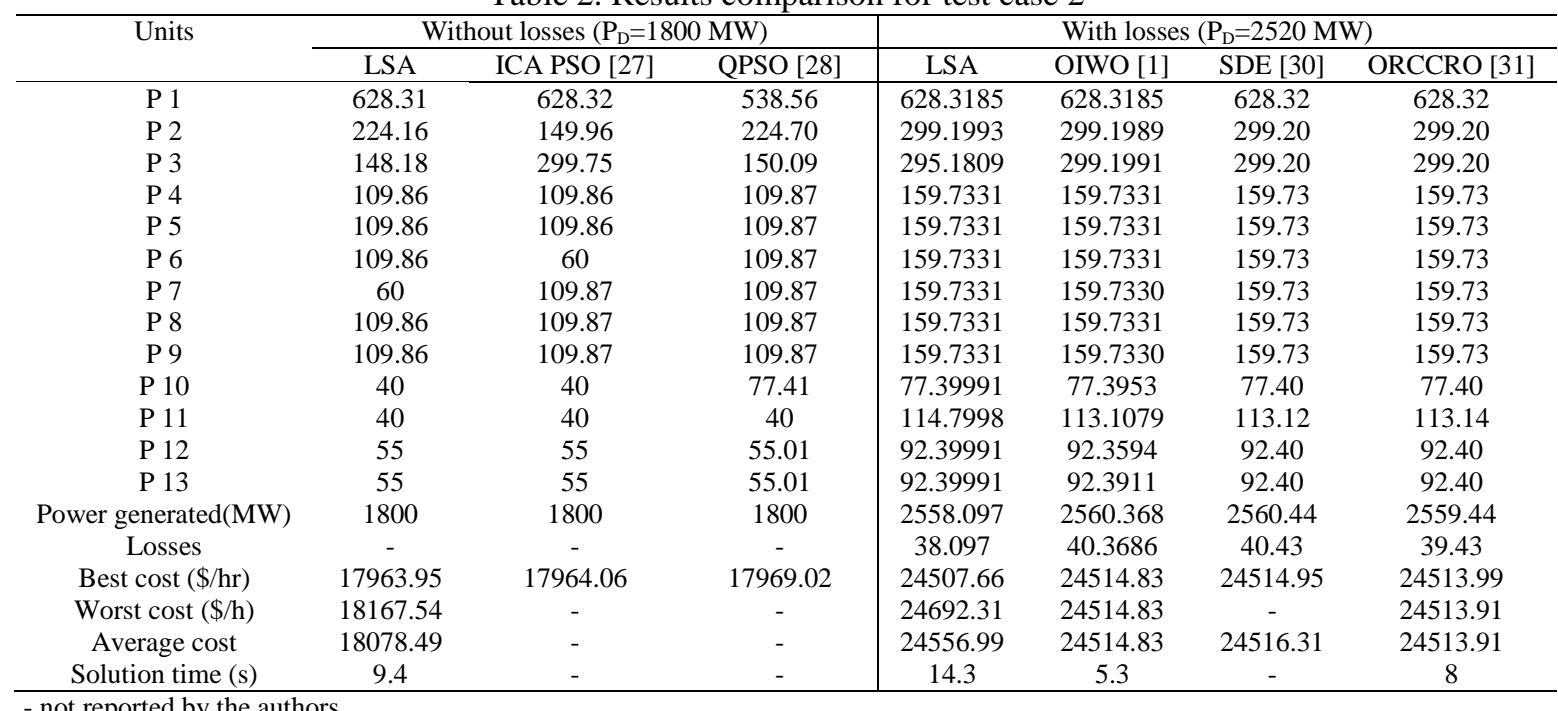

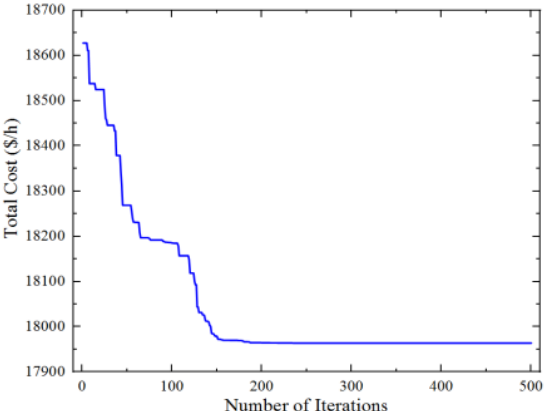

(a)

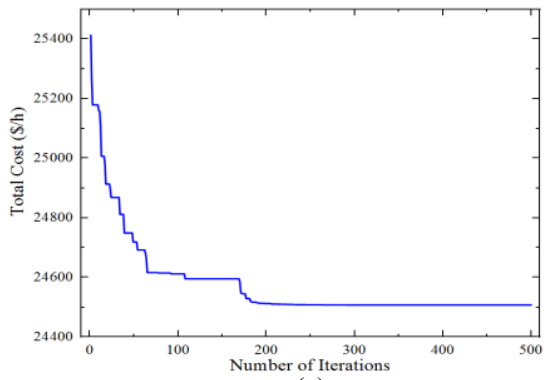

(c)

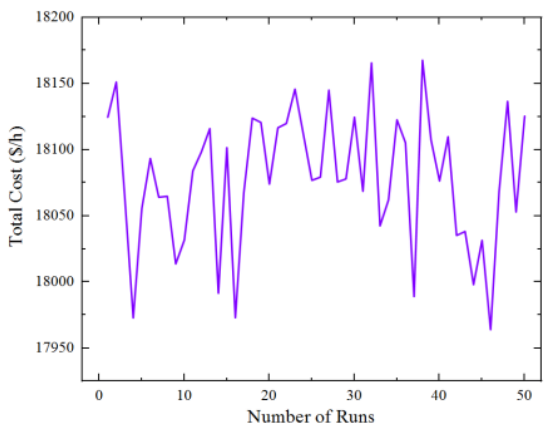

(b)

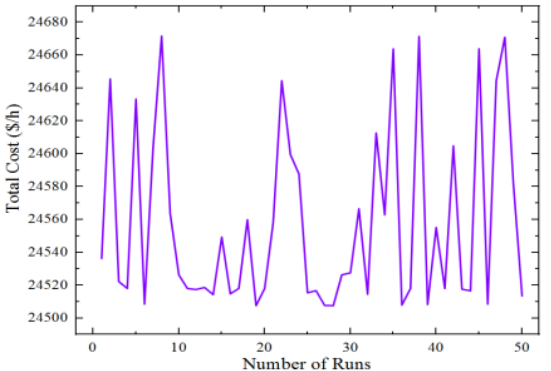

(d)

Figure 3. LSA performance for test case 2. (a) Convergence characteristic without losses (b) Robustness of Cost without losses (c) Convergence characteristic with losses (d) Robustness of Cost with losses 


\section{CONCLUSION}

Minimizing the generation cost and profit increment is a priority in the planning and operation of power systems. Therefore, this paper proposed LSA as a new metaheuristic algorithm for solving the ED problems considering the nonsmooth cost function (valve point effect) and transmission loss. The proposed LSA has been tested on two test cases varied in complexity and size. The optimal results obtained by LSA has been compared with the results obtained by other methods that reported in the literature for the standard 6 and 13 units test system. It found that LSA can produced minimum cost of $925.122 \$ / \mathrm{h}$ and $17963.95 \$ / \mathrm{h}$ for 6 units and 13 units system respectively. The convergence characteristic, robustness, simulation time of proposed LSA are also investigated in this paper for both test systems. The simulation results proved that proposed LSA is outperformed than compared method in term of generation cost. Further investigation can be performed to evaluate LSA performances for minimizing cost and emission level of power generation and other optimization problems.

\section{ACKNOWLEDGEMENTS}

This research was supported by Ministry of Education of Malaysia through Fundamental Research Grant Scheme (FRGS/1/2018/TK04/UTHM/02/17) and partially sponsored by Universiti Tun Hussein Onn Malaysia.

\section{REFERENCES}

[1] A. K. Barisal and R. C. Prusty, "Large scale economic dispatch of power systems using oppositional invasive weed optimization,” Appl. Soft Comput., vol. 29, pp. 122-137, 2015.

[2] M. Hamdi, L. Idomghar, M. Chaoui, and A. Kachouri, "An improved adaptive differential evolution optimizer for non-convex Economic Dispatch Problems," Appl. Soft Comput., vol. 85, p. 105868, 2019.

[3] R. Ramanathan, "Fast Economic Dispatch Based on the Penalty Factors From Newton's Method," IEEE Trans. Power Appar. Syst., vol. PAS-104, no. 7, pp. 1624-1629, 1985.

[4] F. Z. Gherbi and F. Lakdja, "Environmentally constrained economic dispatch via quadratic programming,"in 2011 International Conference on Communications, Computing and Control Applications (CCCA), pp. 1-5, 2011.

[5] G. Irisarri, L. M. Kimball, K. A. Clements, A. Bagchi, and P. W. Davis, "Economic dispatch with network and ramping constraints via interior point methods," IEEE Trans. Power Syst., vol. 13, no. 1, pp. 236-242, 1998.

[6] J. P. Zhan, Q. H. Wu, C. X. Guo, and X. X. Zhou, "Fast \$llambda\$-Iteration Method for Economic Dispatch With Prohibited Operating Zones," IEEE Trans. Power Syst., vol. 29, no. 2, pp. 990-991, 2014.

[7] M. H. Lokman et al., "Multi-verse optimization based evolutionary programming technique for power scheduling in loss minimization scheme," IAES Int. J. Artif. Intell., vol. 8, no. 3, pp. 292-298, 2019.

[8] Z.-X. Liang and J. D. Glover, "A zoom feature for a dynamic programming solution to economic dispatch including transmission losses," IEEE Trans. Power Syst., vol. 7, no. 2, pp. 544-550, 1992.

[9] S. Pothiya, I. Ngamroo, and W. Kongprawechnon, "Ant colony optimisation for economic dispatch problem with non-smooth cost functions," Int. J. Electr. Power Energy Syst., vol. 32, no. 5, pp. 478-487, 2010.

[10] A. Ullah, N. M. Nawi, J. Uddin, S. Baseer, and A. H. Rashed, "Artificial bee colony algorithm used for load balancing in cloud computing: Review," IAES Int. J. Artif. Intell., vol. 8, no. 2, pp. 156-167, 2019.

[11] S. R. Spea, "Economic-emission dispatch problem using firefly algorithm," in 2017 Nineteenth International Middle East Power Systems Conference (MEPCON), 2017, vol. 2018-Febru, pp. 671-676.

[12] M.-K. Baek, J.-B. Park, and K. Y. Lee, "An Improved Attractive and Repulsive Particle Swarm Optimization for Nonconvex Economic Dispatch Problems," IFAC-PapersOnLine, vol. 49, no. 27, pp. 284-289, 2016.

[13] P. M. Joshi and H. K. Verma, "An improved TLBO based economic dispatch of power generation through distributed energy resources considering environmental constraints," Sustain. Energy, Grids Networks, vol. 18, p. 100207, 2019.

[14] A. N. Afandi, I. Fadlika, S. Sendari, H. Miyauchi, G. Fujita, and N. Tutkun, "Comparison of ABC and Genetic Algorithms on Economic Power Dispatch," in 2018 10th International Conference on Information Technology and Electrical Engineering (ICITEE), pp. 40-45, 2018.

[15] P. Zakian and A. Kaveh, "Economic dispatch of power systems using an adaptive charged system search algorithm," Appl. Soft Comput., vol. 73, pp. 607-622, 2018.

[16] N. M. G. K. and S. M. S. D.V. Kiran, "A Comprehensive Review on Optimization Strategies for Combined Economic Emission Dispatch Problem,” Renew. Sustain. Energy Rev., vol. 7, no. 1, pp. 68-74, 2018.

[17] S. M. Moosavian, M. Modiri-Delshad, J. Selvaraj, and N. Abd Rahim, "Solution techniques for optimal power dispatch problems - A review," in 4th IET Clean Energy and Technology Conference (CEAT 2016), pp. 17 (7.)-17 (7.), 2016

[18] T. Niknam, "A new fuzzy adaptive hybrid particle swarm optimization algorithm for non-linear, non-smooth and non-convex economic dispatch problem," Appl. Energy, vol. 87, no. 1, pp. 327-339, 2010.

[19] S. Sayah and A. Hamouda, "A hybrid differential evolution algorithm based on particle swarm optimization for nonconvex economic dispatch problems," Appl. Soft Comput., vol. 13, no. 4, pp. 1608-1619, 2013.

[20] H. Shareef, A. A. Ibrahim, and A. H. Mutlag, "Lightning search algorithm," Appl. Soft Comput., vol. 36, pp. 315333, 2015. 
[21] M. M. Islam, H. Shareef, M. Nagrial, J. Rizk, A. Hellany, and S. N. Khalid, "Performance comparison of various probability gate assisted binary lightning search algorithm," IAES Int. J. Artif. Intell., vol. 8, no. 3, pp. 228-236, 2019.

[22] M. Elsisi and H. Abdelfattah, "New design of variable structure control based on lightning search algorithm for nuclear reactor power system considering load-following operation," Nucl. Eng. Technol., no. xxxx, 2019.

[23] H. Shareef, A. H. Mutlag, and A. Mohamed, "A novel approach for fuzzy logic PV inverter controller optimization using lightning search algorithm," Neurocomputing, vol. 168, pp. 435-453, 2015.

[24] R. Sirjani and E. C. Okonkwo, "A new wind power model using the lightning search algorithm," 13th HONET-ICT Int. Symp. Smart MicroGrids Sustain. Energy Sources Enabled by Photonics IoT Sensors, HONET-ICT 2016, pp. 93-97, 2016.

[25] T. Nadeem Malik, A. ul Asar, M. F. Wyne, and S. Akhtar, "A new hybrid approach for the solution of nonconvex economic dispatch problem with valve-point effects," Electr. Power Syst. Res., vol. 80, no. 9, pp. 1128-1136, 2010.

[26] C. Yaşar and S. Özyön, "A new hybrid approach for nonconvex economic dispatch problem with valve-point effect," Energy, vol. 36, no. 10, pp. 5838-5845, 2011.

[27] P. K. Vitthaladevuni and M.-S. Alouini, "Discussion of 'Economic Load Dispatch - A Comparative Study on Heuristic Optimization Techniques With an Improved Coordinated Aggregation-Based PSO," IEEE Trans. Broadcast., vol. 49, no. 4, pp. 408-408, 2003.

[28] Ke Meng, Hong Gang Wang, ZhaoYang Dong, and Kit Po Wong, "Quantum-Inspired Particle Swarm Optimization for Valve-Point Economic Load Dispatch,” IEEE Trans. Power Syst., vol. 25, no. 1, pp. 215-222, 2010.

[29] L. Dos Santos Coelho and V. C. Mariani, "Combining of Chaotic Differential Evolution and Quadratic Programming for Economic Dispatch Optimization with Valve-Point Effect," IEEE Trans. Power Syst., vol. 21, no. 3 , pp. 1465-1465, 2006.

[30] A. Srinivasa Reddy and K. Vaisakh, "Shuffled differential evolution for large scale economic dispatch," Electr. Power Syst. Res., vol. 96, pp. 237-245, 2013.

[31] K. Bhattacharjee, A. Bhattacharya, and S. H. N. Dey, "Oppositional Real Coded Chemical Reaction Optimization for different economic dispatch problems," Int. J. Electr. Power Energy Syst., vol. 55, pp. 378-391, 2014.

\section{BIOGRAPHIES OF AUTHORS}
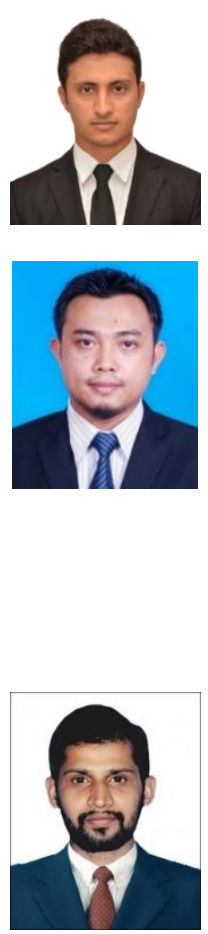

Murad Yahya Nassar received a bachelor's degree in electrical engineering from Universiti Tun Hussein Onn Malaysia, Johor, Malaysia in 2019, where he is pursuing a master's degree in electrical engineering. Her research interest includes power dispatch, renewable energy sources and optimization algorithms.

Dr. Mohd Noor Abdullah received his B.Eng. (Hons) in Electrical Engineering and M. Eng. in Electrical Engineering (Power System) from Universiti Teknologi Malaysia (UTM) in 2008 and 2010 respectively. He also received a Ph.D degree in Electrical Engineering from University of Malaya (UM) in 2014. He has been with Universiti Tun Hussein Onn Malaysia (UTHM) from 2008 to 2014 as a tutor. He is currently as a Lecturer in Department of Electrical Power Engineering, Faculty of Electrical and Electronic Engineering (FKEE), Universiti Tun Hussein Onn Malaysia (UTHM). He also appointed as a head of Green and Sustainable Energy (GSEnergy) Focus Group in FKEE, UTHM. He was a member of Board of Engineer Malaysia. He also a certified of qualified person of SEDA Malaysia Grid Connected Photovoltaic System design. His research interests include electric power dispatch, distributed generation, renewable energy and meta-heuristic optimization techniques.

Asif Ahmed received his B.Eng.in Electronics Engineering and M. Eng. in Electrical Power Engineering from Mehran University Jamshoro, Pakistan (MUET) in 2014 and 2018 respectively. $\mathrm{He}$ is currently pursuing his Ph.D degree in Electrical Engineering from Universiti Tun Hussein Onn Malaysia (UTHM). He has been with Indus University Karachi from Feb-2017 to Jan-2019 as a Lecturer in Department of Electrical technology, Faculty of Science and Technology (FEST). His research interests include power distribution and generation, power electronics and renewable energy. 